\title{
Napoleon Cybulski - a pioneer of neurophysiology in Poland
}

\author{
Rafał Rola
}

\begin{abstract}
Napoleon Nikodem Cybulski (1854-1919), Professor and Head of Physiology Department of the Faculty of Medicine at the Jagiellonian University, was one of the most eminent Polish scientists of the turn of the $20^{\text {th }}$ century. In textbooks on the history of medicine he is rightly credited as the originator of Polish physiology. The Physiology Department headed by Cybulski contributed a number of internationally pioneering discoveries in many areas of physiology. One of the most renowned scientific achievements
\end{abstract}

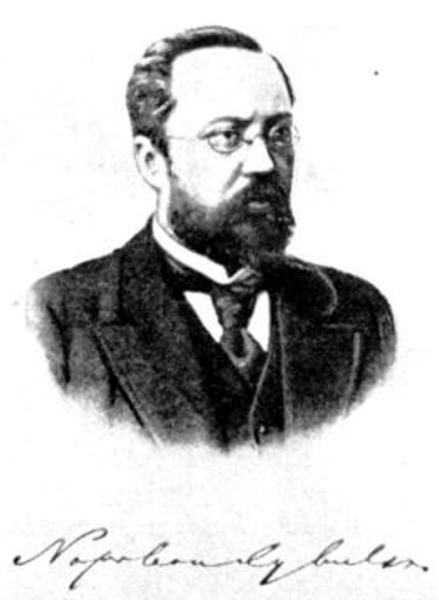

the cerebral cortex (1890), investigations of cortical oscillatory activity (1914) and studies on muscular and nervous excitability (1890-1916)] laid down foundations for the development of a number of methods for nervous system diagnostics: electroencephalography, neurography and sensory evoked potentials. The article outlines Professor Napoleon Cybulski's scientific development, focusing on his achievements in nervous system physiology.

Napoleon Nikodem Cybulski was born in Krzywonosy, in the current terof Professor Cybulski's research team was the discovery, together with Szymonowicz, in 1894-1895, of a substance released from the adrenal cortex into the circulation. The substance, which they called 'nadnerczyna' was found to have a powerful blood pressure elevating effect. The discovery was made independently of studies conducted by the English physiologists Schafer and Oliver who published an article reporting on the influence of adrenal cortical extracts on the circulatory system in 1894. The active substance turned out to be adrenaline, a hormone secreted by the adrenal medulla.

Equally ground-breaking were Professor Cybulski's discoveries and studies on the circulatory system and the physiology of blood flow in blood vessels. Photohemotachometer, a device for measuring blood flow in the vessels designed and constructed by Cybulski, was the most technologically advanced and the most accurate instrument of his day, commonly used by circulatory physiologists. Due to Cybulski's world-class discoveries in the physiology of circulatory and endocrine systems his name is rarely associated with neurology and neurophysiology. Not rightly so. Pioneering research into nervous and muscular excitability and, above all, studies on bioelectrical brain activity, place Cybulski in the rank of international pioneers of neurophysiology and electrophysiology of the nervous system.

Napoleon Cybulski's research output is impressive. Neurophysiological research initiated in the Cracow's Department [recording of sensory evoked potentials in ritory of Lithuania, on 13 September 1854. After graduating with honours from grammar school in 1875, he began his medical studies at the Military Medical Academy in St. Petersburg in that same year. Already in his second year at the Academy, Napoleon Cybulski began research work in Professor Tarkhanov's physiological laboratory. His research interests at that time included physiology of the circulatory system and the effect of body posture on blood pressure and blood flow through the vessels. Similar studies on circulatory system physiology were conducted at the Academy in the same period by Ivan Pavlov. Napoleon Cybulski's first scientific paper on the influence of body posture on the physiology of the circulatory system was published in 1878 . Even before his graduation Cybulski started working as an Assistant in Professor Tarkhanov's laboratory. After graduating from the Military Medical Academy in 1880 Napoleon Cybulski was granted a three-year government scholarship and continued his research as an Assistant in the physiological laboratory. In that period he improved the method for measuring blood flow in the blood vessels and designed his photohemotachometer. Cybulski's photohemotachometer operates by detecting changes in the lateral pressure of blood flow which depend on the blood flow rate. Combination of the manometric technique with a photographic camera made it possible to record very rapid changes in blood flow rate in relation to the phase of the heart's haemodynamic cycle. Cybulski was the first researcher worldwide to 
study variation in blood flow rate depending on the cardiac haemodynamic cycle. Blood flow rate measurements and methodology applied for its investigation were the basis for Cybulski's PhD dissertation on 'Studies on the velocity of blood movement using photohemotachometer' which was defended publicly in St. Petersburg on 13 April 1885. Concurrently, Napoleon Cybulski conducted research into nervous conduction velocity using Deprez's galvanometer and co-authored studies focused on physiology of the vagus nerve, recurrent laryngeal nerve and phrenic nerve. In collaboration with Anrep, he described the function of afferent phrenic nerve fibres in the rhythmogenesis of breathing.

In 1885 , even before the defence of his doctoral dissertation, the Board of the Faculty of Medicine at the Jagiellonian University offered Cybulski the position of Professor of Physiology. Cybulski accepted the offer and went to Cracow after the public defence of his $\mathrm{PhD}$ thesis. The arrival in Cracow marked the beginning of a new period in the young physiologist's personal life and scientific career. The time spent there was the most prolific period in Cybulski's research activity, while research projects and discoveries made in the Cracow's school of physiology gave worldwide fame to Polish science. Napoleon Cybulski set up a research team comprising specialists in different areas of physiology: circulatory and nervous systems, skin and alimentary tract. Many of Professor Cybulski's students then became independent researchers and heads of their own departments, establishing Lvov's school of physiology (Prof. Adolf Beck), Warsaw's school of physiology (Prof. Franciszek Czubalski) and Lvov's school of histology (Prof. Władysław Szymonowicz). The beginnings of Cybulski's scientific work in Cracow were not easy, though. The infrastructure Cybulski found in the Department in Cracow was not up to the standard of other research laboratories of his day. In the initial period, Cybulski embarked on the project of upgrading old facilities in the Collegium Physicum (the main seat of the Physiology Department). Then he invested a lot of effort into administrative activities, applying for funds necessary to build a new facility to house the Department. After three years, following the 1892 decision, a new building of the Collegium Medicum is completed. The edifice, located at 16 Grzegórzecka Street, has been housing the Jagiellonian University's Physiology Department since then. Professor Cybulski devoted the first five years as Head of the Physiology Department to gaining funds for repairs, new equipment and extension of the Department. Despite that, Napoleon Cybulski's scientific career

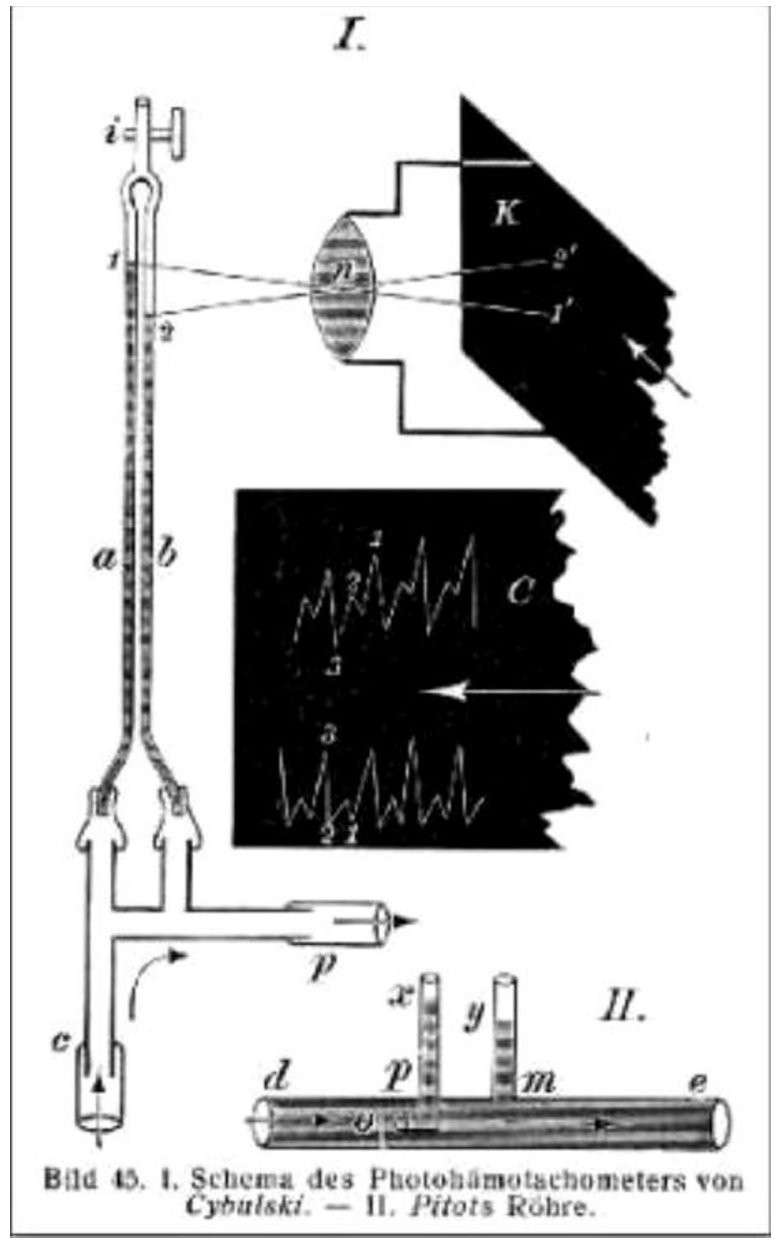

Fig. 1. Diagram illustrating the operation of photohemotachometer

not only did not slow down but gained momentum. He modified his photohemotachometer to adapt it for vein measurements. Adolf Beck, Professor's Assistant, is then able to investigate the effect of vagus nerve stimulation on blood flow through the portal venous bed. In 1888, with Napoleon Cybulski's encouragement and under his supervision, Adolf Beck initiated his own research on variations in the activity of the brain cortex in response to peripheral nerve stimulation. The studies were conducted on macaques and dogs. Electrodes were placed on the cortex to record changes in electric potential triggered by the stimulation of peripheral nerves. After refuting William Horsley's claims that recorded potentials originate from action currents of the skull muscles, they were the first in the world to demonstrate that changes in cortical activity are associated with peripheral nerve stimulation. Analyzing the amplitude 


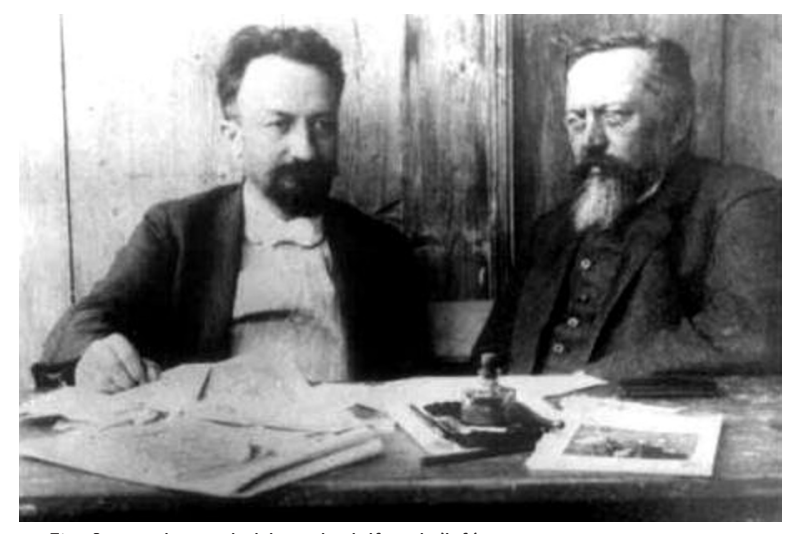

Fig. 2. Napoleon Cybulski and Adolf Beck (left)

of changes in the cortical potential at various sites within the cortex, they mapped cortical regions which are responsible for sensory perception. They also showed that the amplitude of the evoked cortical potential was affected both by the strength and nature of the sensory stimulus, and by the depth of the animal's anaesthesia. Based on these findings, a hypothesis was put forth that activities of the central nervous system are associated with bioelectric activity of cortical neurons. Polish physiologists did not know Richard Caton's earlier investigations of variation in bioelectric activity of the dog's brain cortex during sleep, wakefulness and cessation of functions. Beck's and Cybulski's works were undoubtedly novel in that the two researchers used standardized stimuli to elicit activity and their methodology for mapping the cortical area was based on the amplitude of the evoked electric potential. A completely novel approach was also adopted by Adolf Beck for his research into the bioelectric activity of the frog's spinal cord. Beck presented results of his studies in his $\mathrm{PhD}$ dissertation on 'Identification of locations in the brain and spinal cord using electric phenomena' in 1890.

Napoleon Cybulski resumed his research into the brain's bioelectric activity in 1913-1914, when he recorded changes in bioelectric activity in the form of electric potential fluctuations with a density of $8-10 \mathrm{~Hz}$. The amplitude and frequency of the fluctuations were found to increase during an induced epileptic attack. The observations preceded Berger's discovery of the alpha wave rhythm in the EEG by 15 years. Studies on the brain's bioelectric activity conducted by Cybulski and Beck, along with Caton's research, were the foundation for neurophysiological methods which are currently used in neurology, such as electroencephalography (EEG); sensory, visual and auditory evoked potentials and intra- operative monitoring of eloquent areas during brain tumour resections. The fact that physiologists from Cracow were the first to make these achievements is widely acknowledged around the world. Cybulski's and Beck's works are cited as frequently as Caton's or Berger's.

Another very important area of Napoleon Cybulski's research focused on bioelectric phenomena within the muscles. Most of his time and efforts were devoted to developing a model of propagation of the action potential within the muscle (referred to in Cybulski's time as current, active state or functional state). Cybulski first undertook studies on bioelectric phenomena in the muscles in the early 1890s. At the outset, he improved the methodology for studying bioelectric activity of the muscles. He noted that the shape and properties of recorded electric potentials are largely affected by the nature and duration of the impulse stimulating the nerve or the muscle. Inductors which were typically used at the time had a relatively long discharge time and variable energy of excitation. Cybulski's stimulator (excitator absolutus), designed on the capacitor principle, made it possible to achieve recurrent stimulation of muscles and nerves with a precisely defined electric charge over a specific short time period. The development of a standardized stimulator opened the way for investigations of nervous and muscular excitability and the introduction of concepts of chronaxy and rheobase. In a later period Cybulski studied the properties of resting and action currents in the muscles. He made an attempt at describing the process of initiation and spreading of electric stimuli within the muscle. He described the so-called 'resting current', i.e. the difference between the potential inside the muscle cell and its exterior, currently known as the resting potential of muscle cells. Cybulski made the right prediction and argued his point with contemporary scientists including Hermann (who believed that the potential recorded between the inside of the muscle is only an effect of muscle damage and flow of ions into the cell) claiming that the resting 'current' (potential) is an inherent feature of healthy muscle cells. Professor Cybulski was correct to state that the 'resting current' did not originate inside the cell but in the cell membrane. He also saw the uneven distribution of ions across the cell membrane as a factor triggering the resting potential. He intuitively pointed to the movement of ions as the source of 'action currents'. In order to substantiate his hypotheses, Cybulski created a model of muscle fibre from frog's intestine. Using solutions with different concentrations of ions inside and outside of the intestine, and impregnating the intestinal wall, 
Cybulski created a semi-permeable 'cell membrane'. He recorded differences in potentials between the inside of the 'fibre' and the external environment and stimulated fluctuations of the resting current by means of different ion concentrations. The nature of the 'resting current', Cybulski argued, was to create an 'ionic double layer', with negative ions inside the fibre and positive ions outside. The 'action current' consisted of movement of positive ions along the muscle fibre. Cybulski confirmed the ionic nature of bioelectric phenomena in the muscle in his 1913 study, demonstrating that the first phase of the 'action current', its rate, depended on temperature. Cybulski's observations and hypotheses preceded by over half a century Hodgkin's and Katz's studies into the generation of resting and action potentials within neurons, which is the best evidence for the outstanding scientific intuition of the Cracow physiologist.

Napoleon Cybulski devoted a lot of time to studying properties of the electric potential evoked by stimulation and recorded on the muscle surface. Using two electrodes placed on the muscle surface and connected to a voltmeter, he analyzed changes in 'action current' depending on the strength of the stimulus, distance between the electrodes and composition of the extracellular fluid. He focused in particular on analyzing the rate of increase of the first phase and the time of emergence of the second phase of the 'action current'. The studies would then find practical applications in developing methodologies for electromyographic and electroneurographic investigations.

Napoleon Cybulski's contribution to the development of neurophysiological and electrophysiological methods of examining the nervous system is truly profound. His words, spoken in 1910, in which he expressed hope that 'there will come a time when the electrocardiogram, electromyogram and possibly even electroneurogram will be an equivalent and equally necessary factor for diagnostics as the urine and blood tests are today', turned out to be very fitting.

Napoleon Cybulski's entire scientific output cannot be covered within this short article. However, no description of the life and career of the father of Polish physiology would be complete without a mention of his discovery of adrenal cortical extract with blood pressure elevating properties, which he called 'nadnerczyna', in 1894. Results of the studies were published in 1895, a year after the publication of works by English physiologists who described the same substance, later found to be a hormone and named adrenaline. The English were recognized as the first to make the discovery. At the turn of the $20^{\text {th }}$ century, Napoleon Cybulski set about conducting parallel studies devoted to muscle excitability and physiology of the digestive tract, which initiated the long tradition of the Cracow's school of physiology. He also investigated heat released by working muscles and analyzed electrocardiographic records. The physiology unit headed by Cybulski was a place where numerous works on the physiology of blood coagulation, agglutination and haemolysis of erythrocytes were created.

Napoleon Cybulski's social and didactic activity should not be left unmentioned, either. In the 1880s he gave public lectures popularizing physiological sciences. He originated the idea of establishment of the Faculty of Dentistry alongside the Faculty of Medicine. In the 1890s, together with bacteriologist Odo Bujwid, Cybulski initiated the founding of the first grammar school for girls in Cracow. He was also preoccupied with the problem of nutrition among Galicia's inhabitants and promoted the widespread use of disinfecting agents (milk of lime). He was twice appointed Dean of the Faculty of Medicine and held the function of Rector of the Jagiellonian University for one year. He was an active member of the Polish Academy of Skills (PAU). He actively participated in international physiological congresses. Initially a translator, he authored the first Polish handbook of physiology in collaboration with Adolf Beck in the early $20^{\text {th }}$ century.

Napoleon Cybulski was an inspiration for future generations of Polish physiologists. His students: Szymonowicz, Beck and Czubalski created modern Polish physiology of the first half of the $20^{\text {th }}$ century. Cybulski's pioneering work in neurophysiology and physiology of the circulatory and endocrine systems laid down foundations for contemporary physiology and medicine. 